\title{
WHAT SNU? THE CASE OF THE MISSING SOLAR NEUTRINOS
}

\author{
WILLIAM A. FOWLER
}

California Institute of Technology, Pasadena, Calif., U.S.A.

\begin{abstract}
The current attempts to observe solar neutrinos are reviewed. It is concluded that serious consideration must be given to the possibility that the neutrino flux from the Sun is essentially zero even though we are not yet absolutely forced to this conclusion. Transient effects in the Sun are briefly discussed as a possible explanation for the lack of solar neutrinos.
\end{abstract}

This is a rather late stage in this symposium on the late stages of stellar evolution to call attention to the rather unpleasant fact that there are apparently still severe problems in our understanding of earlier stages and, in particular, of the main-sequence stage. I refer to the case of the missing solar neutrinos, which is still with us. It is my intention in this talk to survey the current situation and future prospects without any elaboration of the background of the case because of lack of time. For an excellent presentation of that background I refer you to a recent review by $R$. W. Kavanagh (1972).

TABLE I

Observations of solar neutrino flux

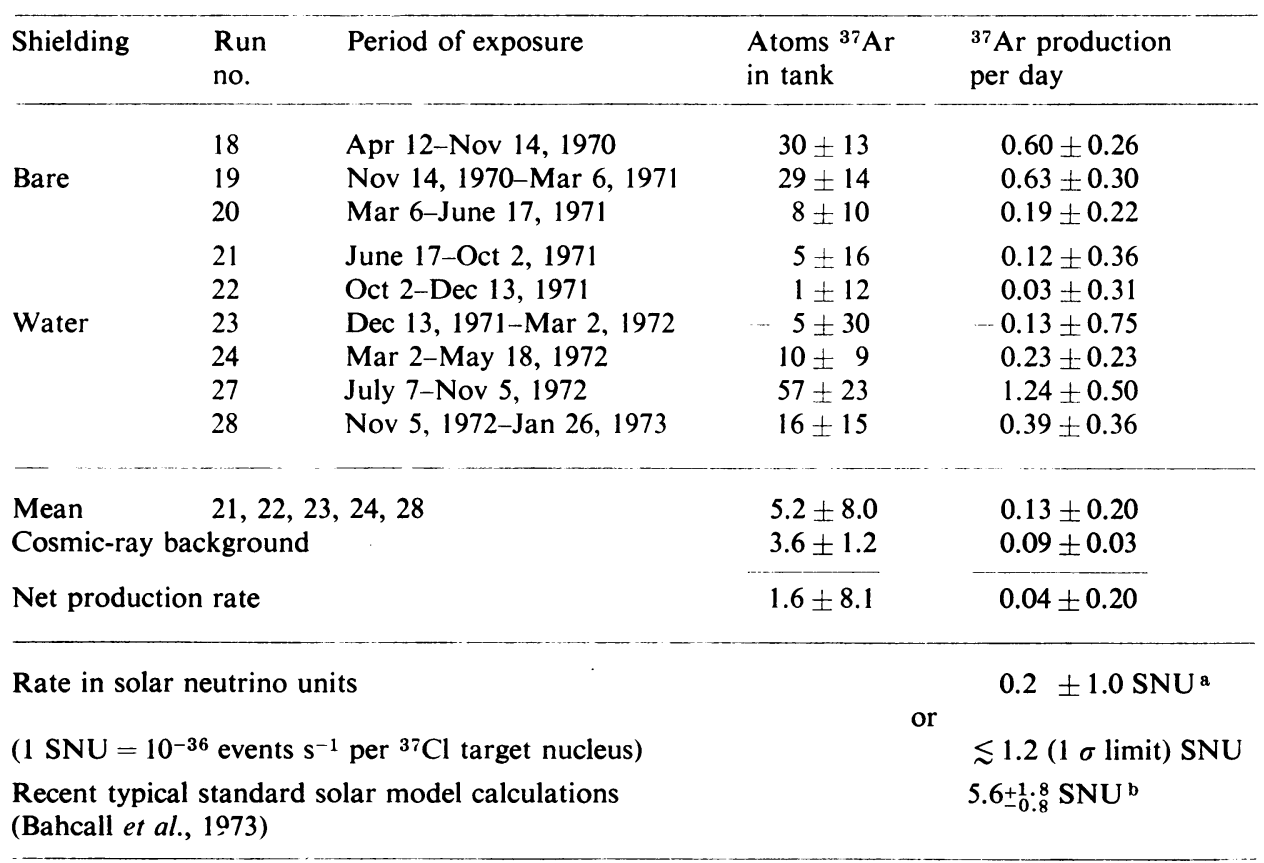

a Two additional runs in 1973 change this to $0.15 \pm 0.75$ SNU with a $1 \sigma$ limit of 0.9 SNU (R. Davis, Jr., private communication).

h Errors quoted due to uncertainties in opacity only. Total standard deviation is approximately $\pm 2.3 \mathrm{SNU}$ (R. Ulrich, private communication). 
Table I presents a summary of recent results obtained by Raymond Davis, Jr. and John C. Evans (1973) at the Brookhaven Neutrino Observatory in the Homestake Gold Mine, Lead, South Dakota, using the Pontecorvo-Alvarez technique of radioactive ${ }^{37} \mathrm{Ar}$ production by neutrino interaction with ${ }^{37} \mathrm{Cl}$. Results are reported for all runs since Davis introduced the 'double window' identification of ${ }^{37} \mathrm{Ar}$ counts using pulse-rise time as well as energy discrimination. The use of the double window and of 'dry run' background tests has enabled Davis to reduce background counts markedly and, in addition, to estimate the accidental background in individual runs. In the tabulated results accidental backgrounds have been subtracted, leading in one case to a net negative number of counts.

The first three runs were made with the tank containing the target ${ }^{37} \mathrm{Cl}$ (in the form of $\mathrm{C}_{2} \mathrm{Cl}_{4}$ ) unshielded from the surrounding rock wall in the mine. There is clearly a background effect - presumably from fast neutrinos produced by $(\alpha, n)$ reactions and spontaneous fission from uranium and thorium contained in the rock. In any case the number of counts decreased when the rock cavity containing the tank was flooded with water producing an effective fast neutron shield by slowing down the neutrons. Run 27 is a marked exception to this statement and Davis and Evans have excluded it from the mean quoted at the bottom of the table "because it probably does not reflect the true neutrino background". They have also excluded the "bare' runs $18,19,20$. Davis and Evans have investigated the possibility that run 27 arose from a supernova event and have calculated that $0.03 M_{\odot}$ equivalent of neutrino radiation with average energy $10 \mathrm{MeV}$ per neutrino at $10 \mathrm{kpc}$ would be required to produce the 57 atoms of ${ }^{37} \mathrm{Ar}$ recovered from the tank (after correction for decay during exposure, etc.).

Not everyone may concur in the exclusion of run 27 but I do.* In the last analysis we can only await the results of further runs and hope that any further high count runs will be accompanied by observable optical, X-ray, or other type events within the exposure period. It is an exciting prospect to say the least.

From the last entries in Table I it will be noted that the $1 \sigma$ upper limit of $1.2 \mathrm{SNU}$ is well below a recent typical standard model calculation by Bahcall et al. (1973) which yielded $5.6_{-0.8}^{+1.8} \mathrm{SNU}$. There have been numerous modifications of the standard solar models which replace the usual virial theorem result for the temperature of the central (c) regions of the Sun

with

$$
k T_{c} \propto G M_{\odot} / R_{\odot}
$$

$k T_{c}+$ rotational terms (Demarque et al., 1973)

+ magnetic field terms (Chitre et al., 1973; Bartenwerfer, 1973) $\propto G M_{\odot} / R_{\odot}$.

In this way $T_{c}$ can be lowered and the very temperature sensitive flux of neutrinos from ${ }^{7} \mathrm{Be}$ and ${ }^{8} \mathrm{~B}$ decays can be markedly reduced. However, in a recent very general

* Including run 27 yields $\sim 1 \ldots 1 \mathrm{SNU}$ or a $1 \sigma$ limit of $\sim 2 \mathrm{SNU}$. 
analysis Ulrich (1974) has shown that it is very difficult to obtain calculated values below 1 SNU when the nuclear processes are treated correctly in detail.*

It is my personal conviction from the results given in Table I that we must now seriously face the possibility that the neutrino flux from the Sun at the earth is essentially zero even though we are not yet absolutely forced to this conclusion. In this case it seems to me that there are only two viable explanations, either the neutrino decays or the Sun decays! For a discussion of neutrino decay and the significant implications for elementary particle physics I refer to Bahcall et al. (1972).

Transient effects in the Sun as an explanation for the lack of solar neutrinos have intrigued me for some years (Fowler, 1969). My most recent discussion (Fowler, 1972, 1973) has been followed by a spate of papers (Dilke and Gough, 1972; Rood, 1972; Ezer and Cameron, 1972; Cameron, 1973) showing that episodic mixing can indeed reduce the solar neutrino flux at the earth to well below $1 \mathrm{SNU}$ and indeed to as low as $0.1 \mathrm{SNU}$. Mixing of new fuels, ${ }^{1} \mathrm{H}$ and ${ }^{3} \mathrm{He}$, into the solar interior results in heating followed by over expansion and cooling to the point where the nuclear reactions are essentially turned off and the Sun shines for a time on its internal store of thermal and gravitational energy. The solar luminosity does decrease markedly and recovers within the Kelvin-Helmholtz time characteristic of the solar core, namely about $5 \times 10^{6} \mathrm{yr}$. In the model calculated by Rood (1972) the Sun was about $20 \%$ more luminous in its steady state about $10^{6} \mathrm{yr}$ ago and it will eventually go through a minimum at about $60 \%$ of its present luminosity and then return to its steady state luminosity. According to Dilke ana Gough (1972) these episodes can occur no more frequently than about once in $250 \times 10^{6} \mathrm{yr}$.

There are a number of important astrophysical and geophysical consequences of this episodic, transient model for the Sun. Terrestrial glaciation and paleoclimatological changes are obvious consequences but there is much controversy about this subject (Öpik, 1952; Emiliani, 1966; Devereux, 1967; Lowenstam and Epstein, 1959). I personally intend to devote considerable study and attention to it in the near future. For the time being one can also point to climatological changes on Mars (Sagan and Young, 1973), to a spread in the main sequence (although this will be small with only one star in 50 deviating at any one time on the episodic model discussed above) and to an increase in the main-sequence lifetime from $\sim 12$ to $\sim 16 \times 10^{9} \mathrm{yr}$ for $M_{\odot}$ (Rood, 1973). Most intriguing to me is the fact that standard solar models imply an increase in the solar luminosity of about $40 \%$ over the Sun's lifetime. This could imply (Sagan and Mullen, 1972) global mean temperatures below the freezing point of sea water less than about $2 \times 10^{9} \mathrm{yr}$ ago, contrary to geologic and paleontological evidence. Rather drastic assumptions about the past composition of the Earth's atmosphere must be made to resolve the problem. The model of Demarque et al. (1973) encounters even greater difficulties in this regard. The episodic, transient mixing model resolves this problem quite simply by requiring a greater luminosity

\footnotetext{
* The oblateness of the Sun produced by these terms is an order of magnitude greater than calculated by the quoted authors and similarly greater than observational limits (R. Rood and R. K. Ulrich. private communication).
} 
for the Sun than in the standard model over all its lifetime except during relatively short periods. Thus, mean global temperatures below that of the freezing temperature of water may never have occurred except for short periods which is consistent with, rather than contrary to, geologic and paleontological evidence.

Another suggestion made by me (Fowler, 1972) and independently by Fetisov and Kopysov (1972) was that a resonance in the reaction ${ }^{3} \mathrm{He}\left({ }^{3} \mathrm{He}, 2 \mathrm{p}\right){ }^{4} \mathrm{He}$ might short circuit the production of the neutrino emitters ${ }^{7} \mathrm{Be}$ and ${ }^{8} \mathrm{~B}$. It would have been a shame if this unexpected loophole in our knowledge of nuclear physics had been the solution of the missing neutrino case but recent experiments have shown that such a resonance and its corresponding excited state in ${ }^{6} \mathrm{Be}$ do not exist (Parker et al., 1973; Dwarakanath, 1974; Halbert et al., 1973).

It will be clear that the case of the missing solar neutrinos is still an exciting scientific detective problem for astronomers, physicists, chemists, and geologists. So, what SNU?

\section{References}

Bahcall, J. N., Cabibbo, N., and Yahil, A.: 1972, Phys. Rev. Letters 28, 316.

Bahcall, J. N., Huebner, W. R., Magee, N. H., Jr., Merts, A. L., and Ulrich, R. K.: 1973, Astrophys. J. 184, 1.

Bartenwerfer, D.: 1973, Astron. Astrophys. 25, 455.

Cameron, A. G. W.: 1973, Rev. Geophys. Space Phys. 11, 505. This paper contains an excellent account of the history of the development of the episodic, transient mixing model of the Sun.

Chitre, S. M., Ezer, D., and Stothers, R.: 1973, Astrophys. Letters 14, 37.

Davis, R., Jr. and Evans, J. C.: 1973, Proceedings of the 13th International Cosmic Ray Conference, Denver, Colorado, August 17-31.

Demarque, P., Mengel, J. C., and Sweigart, A. V.: 1973, Astrophys. J. 183, 997.

Devereux, I.: 1967, New Zealand J. Sci. 10, 988.

Dilke, F. W. W. and Gough, D. O.: 1972, Nature 240, 262.

Dwarakanath, M. R.: 1974, Phys. Rev. C9, 805.

Emiliani, C.: 1966, Science 154, 851.

Ezer, D. and Cameron, A. G. W.: 1972, Nature Phys. Sci. 240, 180.

Fetisov, V. N. and Kopysov, Yu. S.: 1972, Phys. Letters 40B, 602.

Fowler, W. A.: 1969, Contemporary Physics: Trieste Symposium, 370 (International Atomic Energy Agency, Vienna, 1969); Proceedings of Cosmic Ray Studies in Relation to Recent Developments in Astronomy and Astrophysics, 256 (Tata Institute, Bombay, 1969).

Fowler, W. A.: 1972, Nature 238, 24; 1973, 242, 424.

Halbert, M. L., Hensley, D. C., and Bingham, H. G.: 1973, Phys. Rev. C8, 1226.

Kavanagh, R. W.: 1972, in F. Reines (ed.), Cosmology, Fusion and Other Matters, Colorado Associated University Press, Boulder, Colorado, Chapter 10.

Lowenstam, H. A. and Epstein, S.: 1959, Proceedings of the 20th Congreso Geologico Internacional, p. 65.

Öpik, E. J.: 1952, Irish Astron. J. 2, 71.

Parker, P. D., Pisano, D. J., Cobern, M. E., and Marks, G. H.: 1973, Nature Phys. Sci. $241,106$.

Rood, R. T.: 1972, Nature Phys. Sci. 240, 178.

Rood, R. T.: 1973, private communication.

Sagan, C. and Mullen, G.: 1972, Science 177, 52.

Sagan, C. and Young, A. T.: 1973, Nature 243, 459.

Ulrich, R. K.: 1974, Astrophys. J. 188, 369. 